\title{
Self-energy corrections to the MSSM finite-temperature Higgs potential
}

\author{
A. Borisov \\ P.N.Lebedev Physical Institute, Russian Academy of Sciences \\ E-mail: andolboretd.lpi.ru \\ M. Dolgopolov \\ Samara State University \\ E-mail: mvdolgessu.samara.ru

\section{Dubinin} \\ Skobeltsyn Institute of Nuclear Physics, Moscow State University \\ E-mail: dubinindtheory.sinp.msu.ru
}

\begin{abstract}
The one-loop corrections to dimension 2 parameters $\mu_{1,2,12}^{2}$ of the effective high-temperature Higgs potential of the minimal supersymmetric standard model (MSSM) induced by the third generation scalar quarks are calculated. High-temperature and low-temperature approximations can be used for an analysis of temperature evolution for the surface of minima and Higgs boson temperature-dependent mass eigenstates.
\end{abstract}

The XXth International Workshop High Energy Physics and Quantum Field Theory September 24 - October 1, 2011

Sochi, Russia 


\section{Introduction}

Specific properties of the electroweak phase transition in the early universe essential for the following generation of baryonic asymmetry are defined by temperature evolution of Higgs potentials

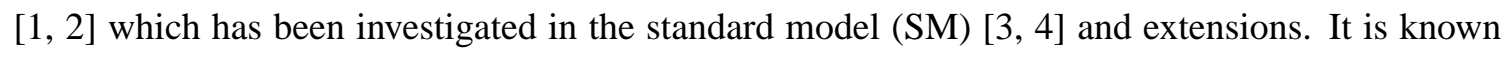
that the simplest $\varphi^{4}$ model leads to the finite-temperature potential [5] (in the high-temperature expansion $m / T<<1$ )

$$
V_{e f f}(v, T)=\frac{1}{2}\left(-\mu^{2}+\frac{1}{4} \lambda T^{2}\right) v^{2}+\frac{1}{4} \lambda v^{4}-\frac{\mu^{2} T^{2}}{24}
$$

which describes the second order phase transition at the critical temperature $T_{c}=2 \mu / \sqrt{\lambda}$. The temperature-dependent minimum is $v^{2}(T)=v^{2}(0)-T^{2} / 4\left(T<T_{c}\right)$ and the thermal mass is $m^{2}(T)=$ $2 \mu^{2}-\lambda T^{2} / 2$. The minimum moves along the line $-v^{4} \lambda / 4$ in the $\left(v, V_{e f f}\right)$ plane. For the tree-level SM Higgs potential $V_{S M}=-\mu^{2} \Phi^{+} \Phi+\lambda\left(\Phi^{+} \Phi\right)^{2}$, where $\Phi^{+}=\frac{1}{\sqrt{2}}\left(\varphi_{1}-i \varphi_{2}, \nu+H-i \varphi_{3}\right)$ minimization condition at the Higgs boson mass $m_{H}^{2}$ has the form

$$
\begin{aligned}
\lambda & =\frac{1}{3 v^{2}}\left(m_{H}^{2}+\mu^{2}\right) \\
\mu^{2} & =\lambda v^{2}
\end{aligned}
$$

which leads to the one-loop high-temperature potential similar to (1.1) whith the only difference that effective $\lambda$ parameter includes gauge boson and top quark contributions: $\lambda \rightarrow \lambda_{S M}=2 \lambda+$ $\frac{g_{2}^{2}+3 g_{1}^{2}}{4}+h_{\text {top }}^{2}$ [6], corresponding to the second order phase transition at the critical temperature $T_{c}=2 v \sqrt{\lambda / \lambda_{S M}}$. In the SM $H$ component of the $S U(2)$ isodoublet coincides with the Higgs mass eigenstate. The situation is more involved in the two-doublet models with the potential ${ }^{1}$

$$
\begin{aligned}
V\left(\Phi_{1}, \Phi_{2}\right) & =-\mu_{1}^{2}\left(\Phi_{1}^{\dagger} \Phi_{1}\right)-\mu_{12}^{2}\left(\Phi_{1}^{\dagger} \Phi_{2}\right)-\mu_{12}^{* 2}\left(\Phi_{2}^{\dagger} \Phi_{1}\right)-\mu_{2}^{2}\left(\Phi_{2}^{\dagger} \Phi_{2}\right)+\lambda_{1}\left(\Phi_{1}^{\dagger} \Phi_{1}\right)^{2}+\lambda_{2}\left(\Phi_{2}^{\dagger} \Phi_{2}\right)^{2} \\
& \left.+\lambda_{3}\left(\Phi_{1}^{\dagger} \Phi_{1}\right)\left(\Phi_{2}^{\dagger} \Phi_{2}\right)+\lambda_{4}\left(\Phi_{1}^{\dagger} \Phi_{2}\right)\left(\Phi_{2}^{\dagger} \Phi_{1}\right)+\frac{\lambda_{5}}{2}\left(\Phi_{1}^{\dagger} \Phi_{2}\right)\left(\Phi_{1}^{\dagger} \Phi_{2}\right)+\frac{\lambda_{5}^{*}}{2}\left(\Phi_{2}^{\dagger} \Phi_{1}\right)\left(\Phi_{2}^{\dagger} \Phi_{1} 1\right) \cdot 3\right) \\
& +\lambda_{6}\left(\Phi_{1}^{\dagger} \Phi_{2}\right)\left(\Phi_{1}^{\dagger} \Phi_{2}\right)+\lambda_{6}^{*}\left(\Phi_{1}^{\dagger} \Phi_{1}\right)\left(\Phi_{2}^{\dagger} \Phi_{1}\right)+\lambda_{7}\left(\Phi_{2}^{\dagger} \Phi_{2}\right)\left(\Phi_{1}^{\dagger} \Phi_{2}\right)+\lambda_{7}^{*}\left(\Phi_{2}^{\dagger} \Phi_{2}\right)\left(\Phi_{2}^{\dagger} \Phi_{1}\right),
\end{aligned}
$$

where doublets of scalar fields

$$
\begin{gathered}
\Phi_{1}=\left(\begin{array}{c}
\phi_{1}^{+}(x) \\
\phi_{1}^{0}(x)
\end{array}\right)=\left(\begin{array}{c}
-i \omega_{1}^{+} \\
\frac{1}{\sqrt{2}}\left(v_{1}+\eta_{1}+i \chi_{1}\right)
\end{array}\right), \\
\Phi_{2}=e^{i \xi}\left(\begin{array}{c}
\phi_{2}^{+}(x) \\
\phi_{2}^{0}(x)
\end{array}\right)=e^{i \xi}\left(\begin{array}{c}
-i \omega_{2}^{+} \\
\frac{1}{\sqrt{2}}\left(v_{2} e^{i \zeta}+\eta_{2}+i \chi_{2}\right)
\end{array}\right)
\end{gathered}
$$

have vacuum expectation values

$$
\left\langle\Phi_{1}\right\rangle=\frac{1}{\sqrt{2}}\left(0, v_{1}\right)^{T}, \quad\left\langle\Phi_{2}\right\rangle=\frac{e^{i \xi}}{\sqrt{2}}\left(0, v_{2} e^{i \zeta}\right)^{T} .
$$

\footnotetext{
${ }^{1}$ in the general case of explicit CP violation 17,8$] \mu_{12}^{2}$ and $\lambda_{5,6,7}$ are complex.
} 
The $S U(2)$ states $\eta_{1,2}$ and $\chi_{1,2}$ are not the mass eigenstates, so mixing angles $\alpha$ and $\beta$ should be introduced

$$
\begin{gathered}
\eta_{1}=-h \sin \alpha+H \cos \alpha, \quad \eta_{2}=h \cos \alpha+H \sin \alpha \\
\chi_{1}=-A \sin \beta+G^{0} \cos \beta, \quad \chi_{2}=A \cos \beta+G^{0} \sin \beta
\end{gathered}
$$

and minimization in the mass basis instead of (1.3) leads to more complicated equations [团]

$$
\begin{aligned}
\lambda_{1} & =\frac{1}{2 v^{2}}\left[\left(\frac{s_{\alpha}}{c_{\beta}}\right)^{2} m_{h}^{2}+\left(\frac{c_{\alpha}}{c_{\beta}}\right)^{2} m_{H}^{2}-\frac{s_{\beta}}{c_{\beta}^{3}} \operatorname{Re} \mu_{12}^{2}\right]+\frac{1}{4}\left(\operatorname{Re} \lambda_{7} \operatorname{tg}^{3} \beta-3 \operatorname{Re} \lambda_{6} \operatorname{tg} \beta\right), \\
\lambda_{2} & =\frac{1}{2 v^{2}}\left[\left(\frac{c_{\alpha}}{s_{\beta}}\right)^{2} m_{h}^{2}+\left(\frac{s_{\alpha}}{s_{\beta}}\right)^{2} m_{H}^{2}-\frac{c_{\beta}}{s_{\beta}^{3}} \operatorname{Re} \mu_{12}^{2}\right]+\frac{1}{4}\left(\operatorname{Re} \lambda_{6} \operatorname{ctg}^{3} \beta-3 \operatorname{Re} \lambda_{7} \operatorname{ctg} \beta\right), \\
\lambda_{3} & =\frac{1}{v^{2}}\left[2 m_{H^{ \pm}}^{2}-\frac{\operatorname{Re} \mu_{12}^{2}}{s_{\beta} c_{\beta}}+\frac{s_{2 \alpha}}{s_{2 \beta}}\left(m_{H}^{2}-m_{h}^{2}\right)\right]-\frac{\operatorname{Re} \lambda_{6}}{2} \operatorname{ctg} \beta-\frac{\operatorname{Re} \lambda_{7}}{2} \operatorname{tg} \beta, \\
\lambda_{4} & =\frac{1}{v^{2}}\left(\frac{\operatorname{Re} \mu_{12}^{2}}{s_{\beta} c_{\beta}}+m_{A}^{2}-2 m_{H^{ \pm}}^{2}\right)-\frac{\operatorname{Re} \lambda_{6}}{2} \operatorname{ctg} \beta-\frac{\operatorname{Re} \lambda_{7}}{2} \operatorname{tg} \beta, \\
\operatorname{Re} \lambda_{5} & =\frac{1}{v^{2}}\left(\frac{\operatorname{Re} \mu_{12}^{2}}{s_{\beta} c_{\beta}}-m_{A}^{2}\right)-\frac{\operatorname{Re} \lambda_{6}}{2} \operatorname{ctg} \beta-\frac{\operatorname{Re} \lambda_{7}}{2} \operatorname{tg} \beta, \\
\mu_{1}^{2} & =\lambda_{1} v_{1}^{2}+\left(\lambda_{3}+\lambda_{4}+\operatorname{Re} \lambda_{5}\right) \frac{v_{2}^{2}}{2}-\operatorname{Re} \mu_{12}^{2} \operatorname{tg} \beta+\frac{v^{2} s_{\beta}^{2}}{2}\left(3 \operatorname{Re} \lambda_{6} \operatorname{ctg} \beta+\operatorname{Re} \lambda_{7} \operatorname{tg} \beta\right), \\
\mu_{2}^{2} & =\lambda_{2} v_{2}^{2}+\left(\lambda_{3}+\lambda_{4}+\operatorname{Re} \lambda_{5}\right) \frac{v_{1}^{2}}{2}-\operatorname{Re} \mu_{12}^{2} \operatorname{ctg} \beta+\frac{v^{2} c_{\beta}^{2}}{2}\left(\operatorname{Re} \lambda_{6} \operatorname{ctg} \beta+3 \operatorname{Re} \lambda_{7} \operatorname{tg} \beta\right) .
\end{aligned}
$$

where (1.8) - (1.12) ensures the diagonalization and (1.13) - (1.14) secures the minimization ${ }^{2}$. Obviously in the case of two background fields $v_{1}(T), v_{2}(T)$ which fix a point on the three-dimensional surface of minima $V_{\text {eff }}\left(v_{1}, v_{2}\right)$ the effective finite-temperature potential cannot be obtained by a redifinition of some parameters in (1.1). Instead of abovementioned SM line of extrema in the two-doublet model the minimum moves along a line on the surface of extrema which can be found by substitution of (1.13) and (1.14) to (1.3). Configuration of the fields (1.7) at any point of the extrema surface $V\left(v_{1}, v_{2}\right)$ must be such that thermal masses $m_{h}(T)$ and $m_{H}(T)$ defined by Eq.(1.8)(1.12) are positively defined. In other words in the process of temperature evolution the matrix of second derivatives of $V\left(\eta_{i}, \chi_{i}\right)$ at the stationary points has only positive eigenvalues which exclude "saddle configurations" in the component fields space.

Note that the two-dimensional picture of thermal evolution in the $v_{1}, v_{2}$ space can be reduced to the one-dimensional picture by a rotation in the $\Phi_{2}, \Phi_{2}$ space to the socalled "Higgs basis" [9], where only $\Phi_{2}$ has nonzero vev $v=\sqrt{v_{1}^{2}+v_{2}^{2}}$. In the Higgs basis the SM-like Higgs potential can be constructed in the decoupling limit $m_{h}<<m_{H}, m_{A}, m_{H^{ \pm}}$(see [10, 11] and the Appendix of [12]). In this proceeding we do not consider the special case of Higgs basis in the two-doublet model.

\section{Temperature-dependent $\mu_{1,2}^{2}$ in the MSSM}

MSSM Higgs sector is a special case of the general two-doublet Higgs sector. At the superparticle mass scale $\lambda_{i}$ parameters are real and expressed by means of the electroweak couplings

\footnotetext{
${ }^{2}$ sometimes (1.8) - (1.14) are mentioned as transformation to the Higgs boson mass basis
} 
$\lambda_{1}=\lambda_{2}=\bar{\lambda}=\frac{g_{1}^{2}+g_{2}^{2}}{8}, \quad \lambda_{3}=\frac{g_{2}^{2}-g_{1}^{2}}{4}, \quad \lambda_{4}=-\frac{g_{2}^{2}}{2}, \quad \lambda_{5}=\lambda_{6}=\lambda_{7}=0 .$. Scalar quarks interaction with MSSM Higgs bosons is defined by the potential

$$
V\left(\Phi_{1}, \Phi_{2}, \widetilde{Q}, \widetilde{U}, \widetilde{D}\right)=\left(\Phi_{i}^{\dagger} \Phi_{j}\right)\left[\Lambda_{i j}^{Q}\left(\widetilde{Q}^{\dagger} \widetilde{Q}\right)+\Lambda_{i j}^{U}\left(\widetilde{U}^{*} \widetilde{U}\right)+\Lambda_{i j}^{D}\left(\widetilde{D}^{*} \widetilde{D}\right)\right]+\bar{\Lambda}_{i j}^{Q}\left(\Phi_{i}^{\dagger} \widetilde{Q}\right)\left(\widetilde{Q}^{\dagger} \Phi_{j}\right) .
$$

where $\widetilde{Q}=\left(\widetilde{u}_{L}, \widetilde{d}_{L}\right)^{T}$ and $\widetilde{U}=\widetilde{u}_{R}^{*}, \widetilde{D}=\widetilde{d}_{R}^{*}$ are left isodoublet and right isosinglet third generation squark fields, respectively. The one-loop finite-temperature contributions to $\mu_{1,2}^{2}$ are defined by

$$
\mu_{i}^{2} \rightarrow \mu_{\beta i}^{2}=\mu_{i}^{2}-\Pi_{i}\left(\beta^{-1}\right)
$$

where

$$
\Pi_{i}\left(\beta^{-1}\right)=\Theta_{i}^{j} f\left(\beta^{-1}, m_{j}\right), f\left(\beta^{-1}, m_{i}\right)=\frac{6}{\pi^{2} \beta^{2}} \int_{0}^{\infty} \frac{x^{2}}{\sqrt{x^{2}+\beta^{2} m_{i}^{2}}} \frac{1}{e^{\sqrt{x^{2}+\beta^{2} m_{i}^{2}}}-1} d x,
$$

and matrix $\Theta_{i}^{j}$ contains the interaction vertices ${ }^{3}$

$$
\left(\Theta_{i}^{j}\right)=\left(\begin{array}{ccc}
h_{D}^{2}-\frac{1}{2} g_{1}^{2} Y_{Q} & -\frac{1}{4} g_{1}^{2} Y_{U} & h_{D}^{2}-\frac{1}{4} g_{1}^{2} Y_{D} \\
h_{U}^{2}+\frac{1}{2} g_{1}^{2} Y_{Q} & h_{U}^{2}+\frac{1}{4} g_{1}^{2} Y_{U} & \frac{1}{4} g_{1}^{2} Y_{D}
\end{array}\right) .
$$

No temperature-dependent contributions to $\mu_{12}^{2}$ are induced. In the high-temperature approximation $m_{i} \beta<1$

$$
f^{h i g h}\left(\beta^{-1}, m_{i}\right)=\frac{1}{\beta^{2}}-\frac{3 m_{i}}{\pi \beta}+O\left(\left(m_{i} \beta\right)^{-1}\right),
$$

while in the low-temperature limit $m_{i} \beta>1$

$$
f^{\text {low }}\left(\beta^{-1}, m_{i}\right)=\frac{6 e^{-m_{i} \beta}}{\pi^{2} m_{i}^{2} \beta^{4}} \sqrt{\frac{\pi}{2 m_{i} \beta}}\left[m_{i}^{3} \beta^{3}+\frac{3}{8} m_{i}^{2} \beta^{2}-\frac{15}{128} m_{i} \beta+\frac{105}{256}+O\left(m_{i}^{-1} \beta^{-1}\right)\right]
$$

so corrections to MSSM mass parameters are

$$
\begin{aligned}
& \Pi_{1}^{h i g h}\left(\beta^{-1}\right)=\Theta_{1}^{j} f^{h i g h}\left(\beta^{-1}, m_{j}\right)=\frac{2 h_{D}^{2}}{\beta^{2}}-\frac{3}{\pi \beta}\left[h_{D}^{2}\left(m_{Q}+m_{D}\right)-\frac{g_{1}^{2}}{6}\left(m_{Q}+m_{D}-2 m_{U}\right)\right], \\
& \Pi_{2}^{h i g h}\left(\beta^{-1}\right)=\Theta_{2}^{j} f^{h i g h}\left(\beta^{-1}, m_{j}\right)=\frac{2 h_{U}^{2}}{\beta^{2}}-\frac{3}{\pi \beta}\left[h_{U}^{2}\left(m_{Q}+m_{U}\right)+\frac{g_{1}^{2}}{6}\left(m_{Q}+m_{D}-2 m_{U}\right)\right]
\end{aligned}
$$

in the high-temperature limit and

$$
\begin{aligned}
\Pi_{1}^{\text {low }}\left(\beta^{-1}\right)= & \Theta_{1}^{j} f^{\text {low }}\left(\beta^{-1}, m_{j}\right)=\frac{6\left(h_{D}^{2}-\frac{1}{6} g_{1}^{2}\right) e^{-m_{Q} \beta}}{\pi^{2} m_{Q}^{2} \beta^{4}} \sqrt{\frac{\pi}{2 m_{Q} \beta}}\left[m_{Q}^{3} \beta^{3}+\frac{3}{8} m_{Q}^{2} \beta^{2}-\frac{15}{128} m_{Q} \beta+\frac{105}{256}\right] \\
& +\frac{2 g_{1}^{2} e^{-m_{U} \beta}}{\pi^{2} m_{U}^{2} \beta^{4}} \sqrt{\frac{\pi}{2 m_{U} \beta}}\left[m_{U}^{3} \beta^{3}+\frac{3}{8} m_{U}^{2} \beta^{2}-\frac{15}{128} m_{U} \beta+\frac{105}{256}\right] \\
& +\frac{6\left(h_{D}^{2}-\frac{1}{6} g_{1}^{2}\right) e^{-m_{D} \beta}}{\pi^{2} m_{D}^{2} \beta^{4}} \sqrt{\frac{\pi}{2 m_{D} \beta}}\left[m_{D}^{3} \beta^{3}+\frac{3}{8} m_{D}^{2} \beta^{2}-\frac{15}{128} m_{D} \beta+\frac{105}{256}\right]
\end{aligned}
$$

\footnotetext{
${ }^{3}$ lower index $i=1,2$ corresponds to $\mu_{1,2}^{2}$ and upper index $j=1,2,3$ corresponds to the squark mass parameters $m_{Q}, m_{U}$ and $m_{D}$.
} 


$$
\begin{aligned}
\Pi_{2}^{\text {low }}\left(\beta^{-1}\right)= & \Theta_{2}^{j} f^{\text {low }}\left(\beta^{-1}, m_{j}\right)=\frac{6\left(h_{U}^{2}+\frac{1}{6} g_{1}^{2}\right) e^{-m_{Q} \beta}}{\pi^{2} m_{Q}^{2} \beta^{4}} \sqrt{\frac{\pi}{2 m_{Q} \beta}}\left[m_{Q}^{3} \beta^{3}+\frac{3}{8} m_{Q}^{2} \beta^{2}-\frac{15}{128} m_{Q} \beta+\frac{105}{256}\right] \\
& +\frac{6\left(h_{U}^{2}-\frac{1}{3} g_{1}^{2}\right) e^{-m_{U} \beta}}{\pi^{2} m_{U}^{2} \beta^{4}} \sqrt{\frac{\pi}{2 m_{U} \beta}}\left[m_{U}^{3} \beta^{3}+\frac{3}{8} m_{U}^{2} \beta^{2}-\frac{15}{128} m_{U} \beta+\frac{105}{256}\right] \\
& +\frac{g_{1}^{2} e^{-m_{D} \beta}}{\pi^{2} m_{D}^{2} \beta^{4}} \sqrt{\frac{\pi}{2 m_{D} \beta}}\left[m_{D}^{3} \beta^{3}+\frac{3}{8} m_{D}^{2} \beta^{2}-\frac{15}{128} m_{D} \beta+\frac{105}{256}\right]
\end{aligned}
$$

in the low-temperature approximation. The one-loop temperature corrections to $\lambda_{i}, i=1, \ldots 7$ have been obtained in [13]. They have weaker dependences on the temperature, so in the case under consideration we neglect correction terms in $\lambda_{i}$.

Substitution of (2) and (2.2) to (1.13) - (1.14), where

$$
\operatorname{Re} \mu_{12}^{2}=\frac{\sin 2 \beta}{2}\left[m_{A}^{2}+\frac{v^{2}}{2}\left(2 \operatorname{Re} \lambda_{5}+\operatorname{Re} \lambda_{6} \cot \beta+\operatorname{Re} \lambda_{7} \tan \beta\right)\right]
$$

includes mass parameter $m_{A}$, dependent on the charged Higgs mass

$$
m_{H^{ \pm}}^{2}=m_{A}^{2}-m_{W^{ \pm}}^{2}-\frac{v^{2}}{2} \operatorname{Re}\left(\lambda_{4}-\lambda_{5}\right),
$$

leads to equations for the one-loop temperature corrections to $\mu_{1,2}^{2}$

$$
\begin{array}{r}
\mu_{1}^{2}-\Pi_{1}\left(\beta^{-1}\right)+m_{A}^{2} \sin ^{2} \beta=\bar{\lambda} v^{2} \cos 2 \beta, \\
\mu_{2}^{2}-\Pi_{2}\left(\beta^{-1}\right)+m_{A}^{2} \cos ^{2} \beta=-\bar{\lambda} v^{2} \cos 2 \beta .
\end{array}
$$

It follows that contours of equilibrium evolution in the $\left(v_{1}, v_{2}\right)$ plane are defined by

$$
\mu_{\beta 1}^{2}+\mu_{\beta 2}^{2}+m_{A}^{2}=0
$$

Mass of the CP-odd state $m_{A}$ does not depend on the temperature, so temperature-dependent terms respect the equation

$$
\Pi_{1}\left(\beta^{-1}\right)+\Pi_{2}\left(\beta^{-1}\right)=0 .
$$

Using the high-temperature approximation (2.5) for contours of minima one can obtain $(T=1 / \beta)$

$$
\tan ^{2} \beta^{h i g h}=\left(\frac{v_{2}}{v_{1}}\right)^{2}=-\frac{m_{t}^{2}}{m_{b}^{2}} \quad \frac{T-\frac{3}{2 \pi}\left(m_{Q}+m_{U}\right)}{T-\frac{3}{2 \pi}\left(m_{Q}+m_{D}\right)}
$$

Note that mixed terms $\sim v_{1} v_{2}$ are cancelled in (2.15), which is important because otherwise not straight lines (2.16) but hyperbolas would be the contours of minima. In such case the equilibrium transition from the symmetric phase to the broken phase would be possible only through some configurations not corresponding to any mass basis of the scalar fields. Using the leading term of the low-temperature approximation (2.6) we get

$$
\tan ^{2} \beta^{\text {low }}=\left(\frac{v_{2}}{v_{1}}\right)^{2}=-\frac{m_{t}^{2}}{m_{b}^{2}} \quad \frac{\sqrt{m_{Q}} e^{-m_{Q} \beta}+\sqrt{m_{U}} e^{-m_{U} \beta}}{\sqrt{m_{Q}} e^{-m_{Q} \beta}+\sqrt{m_{D}} e^{-m_{D} \beta}}
$$


Substitution of the minimization conditions (1.13) - (1.14) to the two-doublet potential gives the surface of minima

$$
V_{\min }^{(\lambda)}\left(v_{1}, v_{2}\right)=-\frac{1}{4}\left(v_{1}^{3} \frac{\partial \Pi_{1}\left(\beta^{-1}\right)}{\partial v_{1}}+v_{2}^{3} \frac{\partial \Pi_{2}\left(\beta^{-1}\right)}{\partial v_{2}}\right)-\frac{\bar{\lambda}}{4}\left(v_{1}^{2}-v_{2}^{2}\right)^{2}
$$

or equivalently

$$
V_{\text {min }}^{(\mu)}\left(v_{1}, v_{2}\right)=-\frac{1}{8}\left(v_{1}^{3} \frac{\partial \Pi_{1}\left(\beta^{-1}\right.}{\partial v_{1}}+v_{2}^{3} \frac{\partial \Pi_{2}\left(\beta^{-1}\right)}{\partial v_{2}}\right)-\frac{1}{4} \mu_{\beta 1}^{2} v_{1}^{2}-\frac{1}{2} \mu_{12}^{2} v_{1} v_{2}-\frac{1}{4} \mu_{\beta 2}^{2} v_{2}^{2},
$$

It follows that in the framework of the MSSM

$$
-\frac{1}{4}\left(v_{1}^{3} \frac{\partial \Pi_{1}\left(\beta^{-1}\right)}{\partial v_{1}}+v_{2}^{3} \frac{\partial \Pi_{2}\left(\beta^{-1}\right)}{\partial v_{2}}\right)=\frac{1}{2} v_{1}^{2} c_{d}^{2}\left(f_{Q}+f_{D}\right)+\frac{1}{2} v_{2}^{2} c_{u}^{2}\left(f_{Q}+f_{U}\right) .
$$

where factors in Yuakwa couplings squared $c_{d}^{2}=\frac{g_{2}^{2}}{2} \frac{m_{d}^{2}}{m_{W}^{2}}$ and $c_{u}^{2}=\frac{g_{2}^{2}}{2} \frac{m_{u}^{2}}{m_{W}^{2}}$ are in front of $\left(v / v_{1}\right)^{2}$ and $\left(v / v_{2}\right)^{2}$, respectively. In the case under consideration the effective parameters $\lambda_{i}$ are temperatureindependent, so the equilibrium matrix for the surface of minima has the form

$$
\Gamma\left(v_{1}, v_{2}\right)=\left\|\begin{array}{cc}
\frac{\partial^{2} V}{\partial v_{1}^{2}} & \frac{\partial^{2} V}{\partial v_{1} \partial v_{2}} \\
\frac{\partial^{2} V}{\partial v_{1} \partial v_{2}} & \frac{\partial^{2} V}{\partial v_{2}^{2}}
\end{array}\right\|=\left\|\begin{array}{cc}
\frac{g_{2}^{2}}{2} \frac{m_{t}^{2}}{m_{W}^{2}}\left(f_{Q}+f_{U}\right)-\bar{\lambda}\left(3 v_{1}^{2}-v_{2}^{2}\right) & 2 \bar{\lambda} v_{1} v_{2} \\
2 \bar{\lambda} v_{1} v_{2} & \frac{g_{2}^{2}}{2} \frac{m_{b}^{2}}{m_{W}^{2}}\left(f_{Q}+f_{D}\right)+\bar{\lambda}\left(v_{1}^{2}-3 v_{2}^{2}\right)
\end{array}\right\|
$$

and the critical temperature is defined by the equation

$$
\left.\operatorname{det} \Gamma\left(v_{1}, v_{2}\right)\right|_{v_{1}=v_{2}=0}=\frac{g_{2}^{4}}{4} \frac{m_{t}^{2} m_{b}^{2}}{m_{W}^{4}}\left[f\left(\beta^{-1}, m_{Q}\right)+f\left(\beta^{-1}, m_{U}\right)\right]\left[f\left(\beta^{-1}, m_{Q}\right)+f\left(\beta^{-1}, m_{D}\right)\right]=0
$$

In the high-temperature approximation two roots of this equation are

$$
T_{c 1}=\frac{3}{2 \pi}\left(m_{Q}+m_{U}\right), \quad T_{c 2}=\frac{3}{2 \pi}\left(m_{Q}+m_{D}\right) .
$$

\section{Summary}

We analyse the temperature evolution of the surface of minima (or the surface of stationary points) of the two-doublet MSSM Higgs potential (1.3), which is defined by substitution of the extrema conditions $\nabla U\left(v_{1}, v_{2}\right)=0$ to the potential. These conditions are expressed symbolicallly by means of linear constraints (1.13), (1.14) on the parameters $\mu_{1}^{1}, \mu_{12}^{2}, \mu_{2}^{2}, \lambda_{i}(\mathrm{i}=1, \ldots 4)$. Any point $\left(v_{1}, v_{2}\right)$ in the background fields space situated on the minima surface and the parameter set $\lambda_{1}, \ldots \lambda_{4}$ define a family of two-doublet potentials with different mass parameters $\mu_{1}^{2}, \mu_{2}^{2}, \mu_{12}^{2}$. At zero temperature $\lambda_{1}, \ldots \lambda_{4}$ are fixed by the MSSM boundary conditions, thereupon $\mu_{1}^{2}, \mu_{2}^{2}, \mu_{12}^{2}$ are unambiguously defined at any given $m_{A}$ and $\tan \beta=v_{2} / v_{1}$. At finite temperature the parameter set $\mu_{1}^{1}, \mu_{2}^{2}, \lambda_{i}$ (i=1,...4) acquires $T$-dependence, leading to evolution of 'thermal masses' and mixing angles owing to a displacement of temperature-dependent vev's $\left(v_{1}, v_{2}\right)$ across the minima surface. For simplicity we neglect the temperature evolution of $\lambda_{i}[13]$ which is weaker than the temperature evolution of $\mu_{1}^{2}, \mu_{2}^{2}$. The parameter $\mu_{12}^{2}$ is temperature-independent because $\Lambda_{i j}$ matrices in the 
squark-Higgs potential (2.1) are diagonal. In the case when both $\mu_{1}^{2}, \mu_{2}^{2}$ and $\lambda_{i}$ are temperaturedependent, equations (1.13)-(1.14) constrain the MSSM parameter space.

At high temperature the two-doublet system is in the symmetric phase with a stable global minimum at $v_{1}=v_{2}=0$, which is assured by derivative terms in (2.18), increasing as $T^{2}$. Conditions of equilibrium thermal evolution must be supplemented by an availability of the scalar boson mass states (Higgs boson mass basis) expressed by (1.8)-(1.12), (2.11). As a consequence of (2.11) one obtains the constraint 2.15) for the one-loop self-energy corrections, which leads to directions of equilibrium thermal evolution (2.16) and (2.17) (in the high-temperature and in the low-temperature approximations, respectively). The critical temperature is defined by zero determinant of the stability matrix, Eq.2.22, two roots of which coincide at degenerate squark mass parameters $m_{Q, U, D}$.

Acknowlegements. Work was partially supported by grants RFBR 10-02-00525-a and NS 1456.2008.2

\section{References}

[1] D.A. Kirzhnits, A.D. Linde, Phys. Lett. B72, 471 (1972); Ann. Phys. 101, 195 (1976) S. Weinberg, Phys. Rev. D9, 3357 (1974)

[2] L. Dolan, R. Jackiw, Phys. Rev. D9, 3320 (1974)

[3] G.W. Anderson and L.J. Hall, Phys. Rev. D45, 2685 (1992).

[4] M.E. Carrington, Phys. Rev. D45, 2933 (1992)

[5] J.R. Espinosa, M. Quiros, F. Zwirner, Phys. Lett. B291, 115 (1992)

[6] J.R. Espinosa, M. Quiros, F. Zwirner, Phys. Lett. B314, 206 (1993)

[7] E. Akhmetzyanova, M. Dolgopolov, M. Dubinin, Phys.Rev. D71 (2005) 075008 (hep-ph/0405264) M.N. Dubinin, A.V. Semenov, Eur. J. Phys. C28, 223 (2003) (hep-ph/0206205)

[8] S.Y. Choi, J.S. Lee, Phys. Rev. D61, 015003 (2000) (hep-ph/9907496)

A. Pilaftsis, C.E.M. Wagner, Nucl. Phys. B553, 3 (1999) (hep-ph/9902371)

D.A. Demir, Phys. Rev. D60, 055006 (1999) (hep-ph/9901389)

[9] M. Sher, Phys. Rept. 179, 273 (1989)

[10] J.R. Espinosa, M. Quiros, F. Zwirner, Phys. Lett. B307, 106 (1993)

[11] A. Brignole, J.R. Espinosa, M. Quiros, F. Zwirner, Phys. Lett. B324, 181 (1994)

[12] E.N. Akhmetzyanova, M.V. Dolgopolov, M.N. Dubinin, Phys. Part. Nucl. 37, 677 (2006)

[13] M.V. Dolgopolov, M.N. Dubinin, E.N. Rykova, J.Mod.Phys. 2, 301 (2011) (arXiv:0901.0524 [hep-ph])

A.O. Borisov, M.V. Dolgopolov, M.N. Dubinin, E.N. Rykova, Phys. Atom. Nucl. 72, 167 (2009) (Yad. Phys. 72, 175 (2009)) 